\title{
Free vibration analysis of composite plates via refined theories accounting for uncertainties
}

\author{
G. Giunta ${ }^{\mathrm{a}, *}$, E. Carrera ${ }^{\mathrm{b}}$ and S. Belouettar ${ }^{\mathrm{a}}$ \\ ${ }^{a}$ Department of Advanced Materials and Structures, Centre de Recherche Public Henri Tudor, \\ Luxembourg-Kirchberg, Luxembourg \\ ${ }^{\mathrm{b}}$ Department of Aeronautic and Space Engineering, Politecnico di Torino, Turin, Italy
}

Received 23 October 2008

Revised 11 January 2010

\begin{abstract}
The free vibration analysis of composite thin and relatively thick plates accounting for uncertainty is addressed in this work. Classical and refined two-dimensional models derived via Carrera's Unified Formulation (CUF) are considered. Material properties and geometrical parameters are supposed to be random. The fundamental frequency related to the first bending eigenmode is stochastically described in terms of the mean value, the standard deviation, the related confidence intervals and the cumulative distribution function. The Monte Carlo Method is employed to account for uncertainty. Cross-ply, simply supported, orthotropic plates are accounted for. Symmetric and anti-symmetric lay-ups are investigated. Displacements based and mixed two-dimensional theories are adopted. Equivalent single layer and layer wise approaches are considered. A Navier type solution is assumed. The conducted analyses have shown that for the considered cases, the fundamental natural frequency is not very sensitive to the uncertainty in the material parameters, while uncertainty in the geometrical parameters should be accounted for. In the case of thin plates, all the considered models yield statistically matching results. For relatively thick plates, the difference in the mean value of the natural frequency is due to the different number of degrees of freedom in the model.
\end{abstract}

Keywords: Free vibration, uncertainty, Monte Carlo Method, refined two-dimensional models, composite plates

\section{Introduction}

Over the last decades, the use of composite materials has increased more and more in aeronautic, space, marine and automotive fields due to the fact that high values of the stiffness-to-weight and the strength-to-weight ratios can be obtained. On the other side, due to their intrinsic anisotropy, the mechanics of composite materials is more complicated than the one of conventional materials. Improvements of the theories that were originally developed for structures made of traditional isotropic materials are required (see, for instance, Carrera [6]). Uncertainty, also, plays a relevant role due to more numerous sources of scattering than conventional materials: constituent mechanical properties, fabrication processes and geometrical parameters. An accurate analysis of composite structures calls, therefore, for the assumption of both higher order models and an effective manner to account for uncertainty. The free vibration analysis of layered plates accounting for uncertainty is addressed in this work. The importance of an accurate analysis is testified, for example, by the fact that closely packed or overlapping natural frequencies are common in aerospace structures. Slightly random variation in the characteristics of the laminates may lead to pronounced effects on the response of the structure. As an example, in the case of the lid of the satellite Corot, the fifth and the seventh modes cross the fourth and the six ones due to the change in structure thickness, see Giunta and

${ }^{*}$ Corresponding author: G. Giunta, Department of Advanced Materials and Structures, Centre de Recherche Public Henri Tudor, 29, av. J. F. Kennedy, L-1855 Luxembourg-Kirchberg, Luxembourg. Tel.: +352 425991 1; Fax: +352 4259 91 555; E-mail: gaetano.giunta@tudor.lu. 
Calvi [12]. Schuëller [39] addressed the need of statistical and probabilistic procedures for a rational treatment of uncertainty and identified the Monte Carlo Method (MCM) as a key procedure in order to use straightforwardly the deterministic models. Among the different methods that account for uncertainty (see Schuëller [40,41] and Isukapalli and Georgopoulos [13]) the MCM is one of the most versatile and powerful. It is often used to validate other statistical procedures as the First Order Perturbation Technique (FOPT), see Caravani and Thomson [4].

To the best of the authors' knowledge, Shinozuka [38] was the first to account for uncertainty in structural dynamics via the MCM. Raj et al. [32] applied it within the finite element method framework to the static analysis of laminates. Singh et al. [44] analysed the stochastic free vibration of thin and thick composite plates via Reddy's [33] refined theory. The shear deformability is modelled via a second order polynomial approximation of the in-plane displacement components. A Navier type solution was adopted. The mean value and the standard deviation of the first five natural frequency were determined via FOPT. Uncertainty was considered to affect only the material properties. FOPT yields valid results only in the case of small dispersion of the stochastic parameters. Yadav and Verma $[49,50]$ investigated the free vibration of composite circular cylindrical shells in terms of mean value and standard deviation. Singh et al. $[42,43]$ studied composite cylindrical and spherical panels. The FOPT method was adopted. The effect of the coefficient of variation of the material properties was investigated. Shaker et al. [37] presented the reliability analysis of the free vibration of composite plates via the first- and second-order reliability methods and the stochastic finite element method.

As far as deterministic refined theories are concerned, Kheider and Reddy [17] modelled the transverse shear deformation via a second order polynomial approximation of the in-plane displacement components. A Levy type solution and Hamilton's principle were adopted. Messina [21] developed two higher order theories for the free vibration analysis of cross-ply and angle-ply laminates under cylindrical bending. The first model is a displacement based theory in which a suitable orthonormal polynomial base was used in order to satisfy the mechanical boundary conditions at plate top and bottom. The second one was based on Reissner's mixed variational theorem (see Reissner [34]) in order to ensure a priori the continuity of the out-of-plane stresses through the thickness. Aydogdu and Timarci [2] used a simplified version of the displacement based model by Messina in order to investigate the free vibration behaviour of cross-ply laminated plates subjected to different sets of boundary conditions. Matsunaga [19] and Kant and Swaminathan [16] formulated kinematic hypotheses that accounted also for the transverse deformability. Ganapathi and Makhecha [11] introduced a zig-zag function such that the displacement components are $C^{0}$ class through the thickness.

In this paper, Carrera's Unified Formulation (CUF), see Carrera [8], is addressed and adopted in order to derive classical and highly accurate two-dimensional models. The MCM is adopted in order to account for uncertainties affecting both composite material properties and plate geometry. In such a manner, an accurate description of composite plates mechanics is coupled to a very effective stochastic procedure. The fundamental natural frequency related to the bending eigenmode characterised by half wave along the in-plane directions is statistically determined in terms of mean value, standard deviation and related intervals of confidence. A confidence level of 0.95 is assumed. The Cumulative Distribution Function (CDF) is also determined. A Navier type solution is adopted. Simply supported, cross-ply laminates are, therefore, investigated. The assumption of a closed form solution tackles the drawback of the MCM regarding the computational effort.

CUF is adopted since it allows to formulate a wide number of two-dimensional models on the basis of: 1the choice of the main unknowns; 2- the order of approximation; 3- the approach to the approximation. Refined two-dimensional models can be formulated according to the required level of accuracy. Transverse shear and normal deformability as well as rotatory inertia can be accounted for. $C^{0}$ requirements (see Carrera [5]) can be fulfilled for both displacement and out-of-plane stress components. Classical Theories (CTs) based on Cauchy's [9], Poisson's [28], Kirchhoff's [18], Reissner's [35] and Mindlin's [24] can be also obtained via CUF as particular cases.

Some applications of CUF models to the deterministic free vibration analysis can be found in Carrera $[8,7]$. Numerical results were assessed via comparison toward three-dimensional solutions. CUF and related classical and refined theories are addressed and assessed in section 2. MCM is discussed briefly in section 3. Numerical results are presented and discussed in section 4 . The last section is dedicated to the conclusions. 


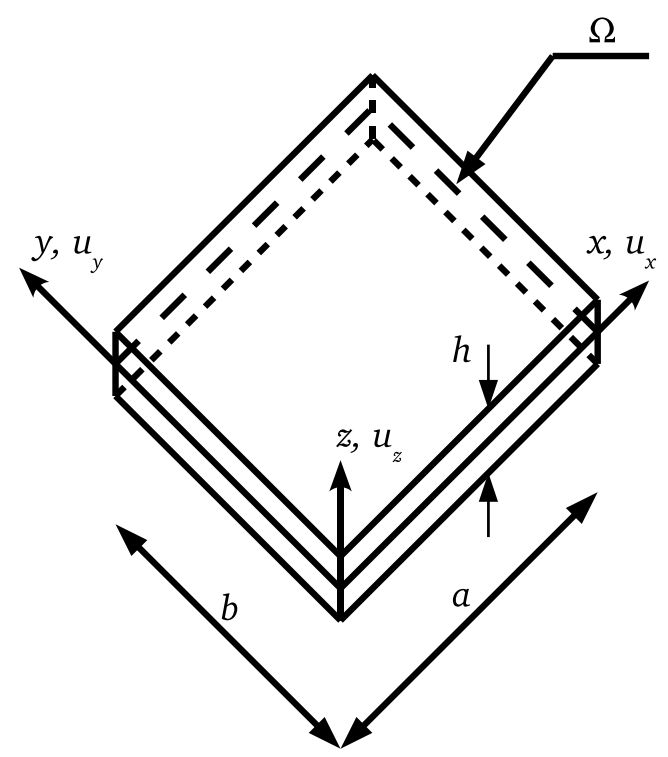

Fig. 1. Plate's geometry and reference system.

\section{Refined plate theories via Carrera's unified formulation}

The term plate stands for a structure without curvature whose two dimensions are dominant with respect to the third one perpendicular to them. The negligible dimension is the thickness of the structure and it identifies the $z$ axis. The predominant dimensions, $a$ and $b$, represent the sides of the plate. They identify the in-plane $x$ and $y$ directions and the reference plane $\Omega$, see Fig. 1. The coordinates are bounded such that: $0 \leqslant x \leqslant a, 0 \leqslant y \leqslant b$, $-h / 2 \leqslant z \leqslant h / 2$. The notation for the displacement field is:

$$
\mathbf{u}^{T}=\left\{\begin{array}{lll}
u_{x} & u_{y} & u_{z}
\end{array}\right\}
$$

' $T$ ' is the transposition operator. Stress $\sigma$ and strain $\epsilon$ vector components are grouped into in-plane terms $\sigma_{p}, \epsilon_{p}$ :

$$
\sigma_{p}^{T}=\left\{\begin{array}{lll}
\sigma_{x x} & \sigma_{y y} & \sigma_{x y}
\end{array}\right\}, \epsilon_{p}^{T}=\left\{\begin{array}{lll}
\epsilon_{x x} & \epsilon_{y y} & \epsilon_{x y}
\end{array}\right\}
$$

and out-of-plane terms $\sigma_{n}, \epsilon_{n}$ :

$$
\sigma_{n}^{T}=\left\{\sigma_{x z} \sigma_{y z} \sigma_{z z}\right\}, \epsilon_{n}^{T}=\left\{\epsilon_{x z} \epsilon_{y z} \epsilon_{z z}\right\}
$$

Being the plate made of linear elastic orthotropic materials, generalised Hooke's law holds:

$$
\begin{aligned}
\sigma_{p} & =\widetilde{\mathbf{C}}_{p p} \epsilon_{p}+\widetilde{\mathbf{C}}_{p n} \epsilon_{n} \\
\sigma_{n} & =\widetilde{\mathbf{C}}_{n p} \epsilon_{p}+\widetilde{\mathbf{C}}_{n n} \epsilon_{n}
\end{aligned}
$$

The terms $\left\{\widetilde{\mathbf{C}}_{i j}: i, j=p, n\right\}$ in Eq. (4) are the material stiffness coefficients. For the sake of brevity, they are not reported here. They can be found in Carrera [8]. Under the hypothesis of small displacements, linear strains-displacements relations can be adopted:

$$
\begin{aligned}
\epsilon_{p} & =\mathbf{D}_{p} \mathbf{u} \\
\epsilon_{n} & =\left(\mathbf{D}_{n \Omega}+\mathbf{D}_{n z}\right) \mathbf{u}
\end{aligned}
$$

$\mathbf{D}_{p}, \mathbf{D}_{n \Omega}$ and $\mathbf{D}_{n z}$ matrices are array differential operators:

$$
\mathbf{D}_{p}=\left[\begin{array}{ccc}
\frac{\partial}{\partial x} & 0 & 0 \\
0 & \frac{\partial}{\partial y} & 0 \\
\frac{\partial}{\partial y} & \frac{\partial}{\partial x} & 0
\end{array}\right], \mathbf{D}_{n \Omega}=\left[\begin{array}{ccc}
0 & 0 & \frac{\partial}{\partial x} \\
0 & 0 & \frac{\partial}{\partial y} \\
0 & 0 & 0
\end{array}\right], \mathbf{D}_{n z}=\left[\begin{array}{ccc}
\frac{\partial}{\partial z} & 0 & 0 \\
0 & \frac{\partial}{\partial z} & 0 \\
0 & 0 & \frac{\partial}{\partial z}
\end{array}\right]
$$


A considerable number of plate models can be formulated and hierarchically classified on the basis of: 1- the choice of the main unknowns or, equivalently, of the variational statement; 2 - the polynomial expansion order along the thickness direction; 3- the way the approximation is imposed (either above the whole laminate or lamina by lamina).

\subsection{The variational statements}

In order to derive the problem governing equations, two variational statements are considered: D'Alembert's Principle (DP) and Reissner's Mixed Variational Theorem (RMVT). A model will furnish the displacement components if DP is adopted:

$$
\int_{\Omega} \int_{h}\left(\delta \epsilon_{p G}^{T} \sigma_{p H}+\delta \epsilon_{n G}^{T} \sigma_{n H}\right) d z d \Omega=\int_{\Omega} \int_{h} \rho \delta \mathbf{u} \ddot{\mathbf{u}} d z d \Omega
$$

Subscript ' $G$ ' means that the strain components are obtained by means of derivation of the displacements field (see Eq. (5)). ' $H$ ' stands for variable computed via material constitutive equations, that is, generalised Hook's law in Eq. (4). The material density is represented by $\rho$. Dots in position of superscript stand for derivation with respect to time. In the case of plate models based on RMVT:

$$
\int_{\Omega} \int_{h}\left[\delta \epsilon_{p G}^{T} \sigma_{p H}+\delta \epsilon_{n G}^{T} \sigma_{n M}+\delta \sigma_{n M}^{T}\left(\epsilon_{n G}-\epsilon_{n H}\right)\right] d z d \Omega=\int_{\Omega} \int_{h} \rho \delta \mathbf{u} \ddot{\mathbf{u}} d z d \Omega
$$

both the displacement and the out-of-plane stress components are assumed as main unknowns. Subscript ' $M$ ' indicates that the stress components are a priori modelled. These models can describe very accurately the peculiar behaviour of composite laminates due to the transverse material discontinuity.

\subsection{The polynomial expansion order}

In the case of two-dimensional models, the generic main unknown component $f$ is approximated in the following manner:

$$
f(x, y, z ; t)=F_{\tau}(z) g_{\tau}(x, y) h(t) \quad \tau=0,1, \ldots, N
$$

in which, according to Einstein's notation, repeated subscript $\tau$ stands for summation. Each term $F_{\tau}$ is an axiomatically postulated function of $z$. In the literature, several functions were proposed to approximate the variation through the thickness. Touratier [48] assumed sine functions, while hyperbolic sine and cosine functions were adopted by Soldatos [45]. Within CUF, $N$-order polynomial functions are considered. The type of the polynomial function depends on the manner the approximation is imposed: at laminate level or at lamina one. The maximum polynomial order is considered to be four. Function $h(t)$ is:

$$
h(t)=e^{i \omega_{m n} t}
$$

in which $i=\sqrt{-1}$ and $\omega_{m n}$ is the natural frequency of the eigenmode characterised by $m$ and $n$ half waves along the directions $x$ and $y$, respectively. Time variable is symbolised by $t$. Each Function $g_{\tau}(x, y)$ represents the variation of the problem unknown toward the in-plane coordinates. According to Navier's solution, it is a combination of sine and cosine functions that depends on the unknown component:

$$
\begin{aligned}
& \left(u_{x}, \sigma_{x z}\right)=\left(\widetilde{u}_{x}(z), \widetilde{\sigma}_{x z}(z)\right) \cos \left(\frac{m \pi}{a} x\right) \sin \left(\frac{n \pi}{b} y\right) e^{i \omega_{m n} t} \\
& \left(u_{y}, \sigma_{y z}\right)=\left(\widetilde{u}_{y}(z), \widetilde{\sigma}_{y z}(z)\right) \sin \left(\frac{m \pi}{a} x\right) \cos \left(\frac{n \pi}{b} y\right) e^{i \omega_{m n} t} \\
& \left(u_{z}, \sigma_{z z}\right)=\left(\widetilde{u}_{z}(z), \widetilde{\sigma}_{z z}(z)\right) \sin \left(\frac{m \pi}{a} x\right) \sin \left(\frac{n \pi}{b} y\right) e^{i \omega_{m n} t}
\end{aligned}
$$

$\left(\widetilde{u}_{x}, \widetilde{u}_{y}, \widetilde{u}_{z}\right)$ and $\left(\widetilde{\sigma}_{x z}, \widetilde{\sigma}_{y z}, \widetilde{\sigma}_{z z}\right)$ are the maximal amplitudes of displacement and out-of-plane stress components, respectively. 


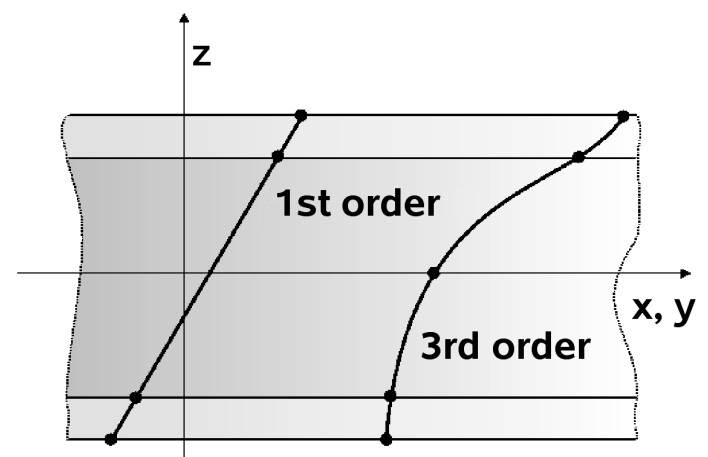

Fig. 2. First- and third-order polynomial approximations for the Equivalent Single Layer approach.

\subsection{Laminate and lamina by lamina approximations}

In the case of Equivalent Single Layer (ESL) models, an axiomatic approximation of problem main unknowns is continuously defined across the whole laminate thickness. Transverse material anisotropy is accounted for via average mechanical properties in terms of stiffness and density. For ESL models, each polynomial term $F_{\tau}$ in Eq. (9) is an element of the classical polynomial base:

$$
F_{\tau}(z)=z^{\tau} \quad \tau=0,1, \ldots, N
$$

The first- and third-order ESL polynomial approximations are shown in Fig. 2. In the case of classical models, $N$ is equal to one for the in-plane displacement components while the transverse displacement is constant. Higher order ESL models accounting for transverse shear and normal deformability and rotatory inertia can be formulated assuming $N$ higher than one. The mechanics of composites may be not accurately described by an ESL approach in the case of thick plates or high degree of anisotropy. A Layer-Wise (LW) description (that is, layer by layer) should be rather considered. According to the LW approach, Eq. (9) holds at layer level:

$$
f^{k}(x, y, z ; t)=F_{\tau}\left(\zeta_{k}(z)\right) g_{\tau}^{k}(x, y) h(t) \quad \tau=0,1, \ldots, N k=1,2, \ldots, N_{l}
$$

being $N_{l}$ the total number of layers and $\zeta_{k}(z)$ a $k$-layer local, dimensionless coordinate along the thickness such that $-1 \leqslant \zeta_{k} \leqslant 1$. The imposition of compatibility and equilibrium at layer interfaces ensures the integrity of the plate. Each $F_{\tau}\left(\zeta_{k}(z)\right)$ function is considered to be a linear combination of Legendre's polynomials:

$$
\begin{array}{r}
F_{0}\left(\zeta_{k}\right)=\frac{P_{0}\left(\zeta_{k}\right)-P_{1}\left(\zeta_{k}\right)}{2}, \quad F_{1}\left(\zeta_{k}\right)=\frac{P_{0}\left(\zeta_{k}\right)+P_{1}\left(\zeta_{k}\right)}{2}, \\
F_{r}\left(\zeta_{k}\right)=\left[P_{r}\left(\zeta_{k}\right)-P_{r-2}\left(\zeta_{k}\right)\right] r=2,3, \ldots, N
\end{array}
$$

Each $P_{\tau}\left(\zeta_{k}\right)$ is defined by means of the following recursive formula:

$$
\begin{gathered}
P_{0}\left(\zeta_{k}\right)=1, P_{1}\left(\zeta_{k}\right)=\zeta_{k} \\
(n+1) P_{n+1}\left(\zeta_{k}\right)=(2 n+1) \zeta_{k} P_{n}\left(\zeta_{k}\right)-n P_{n-1}\left(\zeta_{k}\right) \quad n=1,2, \ldots, N-1
\end{gathered}
$$

LW models yield $C^{0}$ class functions with respect to the through-the-thickness coordinate (see Fig. 3). They are intrinsically capable to describe the through-the-thickness zig-zag variation of displacements and out-of-plane stresses peculiar of the mechanics of composites. ESL theories are based on $C^{\infty}$ class functions and they are not able to predict the zig-zag variation. This can be retrieved via the adoption of a zig-zag function. Murakami's zig-zag function (see Murakami [25]), is adopted within CUF. An historical review of zig-zag functions can be found in Carrera [6]. In the case of the Murakami’s function, the postulated kinematic field is:

$$
\begin{aligned}
& F_{\tau}(z)=z^{\tau} \tau=0,1, \ldots, N \\
& F_{N+1}(z)=(-1)^{k} \zeta_{k}
\end{aligned}
$$

Third-order zig-zag ESL model is presented in Fig. 4. 


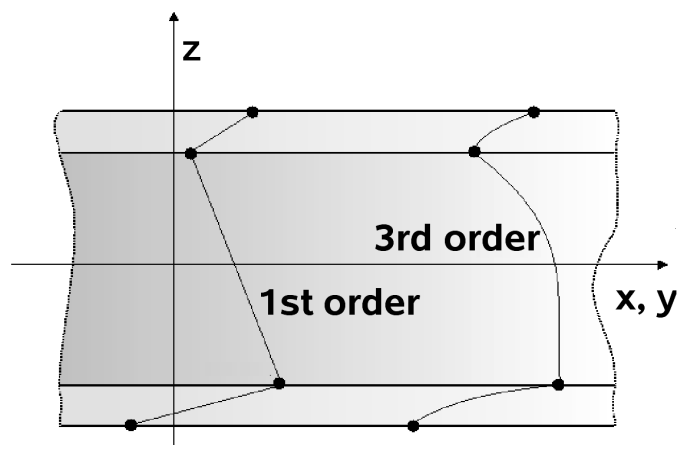

Fig. 3. First- and third-order polynomial approximations for the Layer-Wise approach.

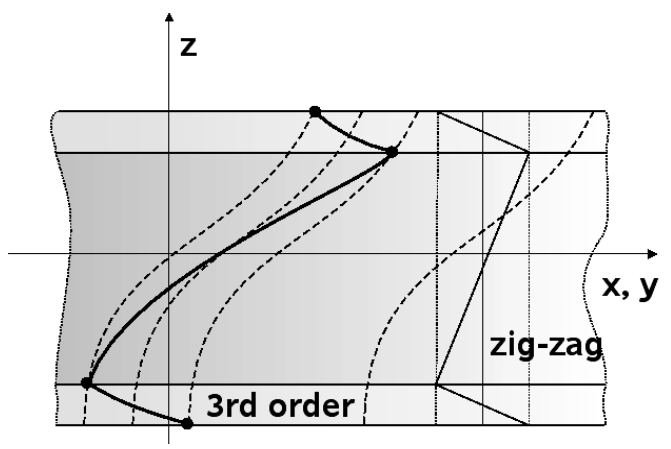

Fig. 4. Third-order polynomial approximation with Murakami’s zig-zag function for the Equivalent Single Layer approach.

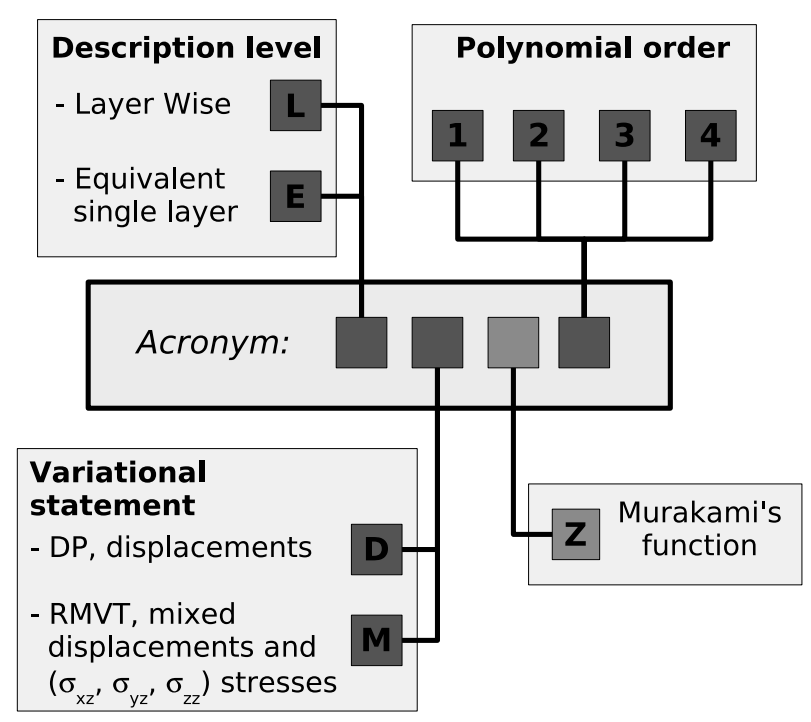

Fig. 5. Acronym system.

\subsection{The acronym system and the unified notation}

A way to address CUF two-dimensional theories is here presented. It is based on the acronym system shown in Fig. 5. The first letter, either 'L' or 'E', specifies whether a LW or an ESL approach is assumed. The second letter indicates the variational statement: 'D' for DP or ' $M$ ' in the case of RMVT. The number represents the polynomial 
approximation order. The third letter, whenever present, is ' $Z$ ' to indicate that Murakami's function has been used. Some examples of CUF theories follow. A generic EDN model is an ESL, displacement based theory with a $N$ order polynomial approximation:

$$
\begin{aligned}
& u_{x}=u_{x 0}+u_{x 1} z+u_{x 2} z^{2}+\ldots+u_{x N} z^{N} \\
& u_{y}=u_{y 0}+u_{y 1} z+u_{y 2} z^{2}+\ldots+u_{y N} z^{N} \\
& u_{z}=u_{z 0}+u_{z 1} z+u_{z 2} z^{2}+\ldots+u_{z N} z^{N}
\end{aligned}
$$

In a vectorial form:

$$
\mathbf{u}=F_{0} \mathbf{u}_{0}+F_{1} \mathbf{u}_{1}+\ldots+F_{N} \mathbf{u}_{N}=F_{\tau} \mathbf{u}_{\tau} \quad \tau=0,1, \ldots, N
$$

being $F_{\tau}=z^{\tau}$ and $\mathbf{u}_{\tau}=\mathbf{u}_{\tau}(x, y ; t)$. The kinematic field of a generic EDZN theory is:

$$
\begin{aligned}
& u_{x}=u_{x 0}+u_{x 1} z+u_{x 2} z^{2}+\ldots+u_{x N} z^{N}+(-1)^{k} \zeta_{k} u_{x N+1} \\
& u_{y}=u_{y 0}+u_{y 1} z+u_{y 2} z^{2}+\ldots+u_{y N} z^{N}+(-1)^{k} \zeta_{k} u_{y N+1} \\
& u_{z}=u_{z 0}+u_{z 1} z+u_{z 2} z^{2}+\ldots+u_{z N} z^{N}+(-1)^{k} \zeta_{k} u_{z N+1}
\end{aligned}
$$

In a compact notation:

$$
\mathbf{u}=F_{0} \mathbf{u}_{0}+F_{1} \mathbf{u}_{1}+\ldots+F_{N} \mathbf{u}_{N}+F_{N+1} \mathbf{u}_{N+1}=F_{\tau} \mathbf{u}_{\tau} \quad \tau=0,1, \ldots, N, N+1
$$

For LMN theories, a $N$-order polynomial approximation is assumed layer by layer for both displacement and out-of-plane stress components. Its unified vectorial notation follows:

$$
\begin{aligned}
\mathbf{u}^{k} & =F_{0} \mathbf{u}_{0}^{k}+F_{1} \mathbf{u}_{1}^{k}+\ldots+F_{N} \mathbf{u}_{N}^{k}=F_{\tau} \mathbf{u}_{\tau}^{k} \\
\sigma^{k} & =F_{0} \sigma_{0}^{k}+F_{1} \sigma_{1}^{k}+\ldots+F_{N} \sigma_{N}^{k}=F_{\tau} \sigma_{\tau}^{k}
\end{aligned} \quad \tau=0,1, \ldots, N ; k=1,2, \ldots, N_{l}
$$

Each term $F_{\tau}^{k}$ is addressed in Eq. (14). The considered theories can be all unified considering that classical theories such as Classical Lamination Theory and First Order Shear Deformation Theory (FSDT) are particular cases of ESL higher order models. These latter can be regarded as a particular case of LW models in which the number of layers is equal to the unit and the through-the-thickness polynomial approximation is carried out via the classical polynomial base. This unifying idea leads to the assumption of the common notation presented in Eqs (18), (20) and (21) that allows to derive the governing equations straightforwardly.

\subsection{The governing equations}

Although in the numerical analysis both DP- and RMVT-based models will be used, for the sake of brevity, governing equations and related boundary conditions are here derived via DP only. The extension to the RMVT case is straightforward and it can be found in Carrera [8].

In the case of a LW approach, DP variational statement presented in Eq. (7) becomes:

$$
\iint_{\Omega_{k}} \delta \epsilon_{p}^{k T} \sigma_{p}^{k}+\delta \epsilon_{n}^{k T} \sigma_{n}^{k} d z_{k} d \Omega_{k}=\int_{\Omega_{k}} \int_{h_{h}} \rho \delta \mathbf{u}^{k} \ddot{\mathbf{u}}^{k} d z_{k} d \Omega_{k} k=1,2, \ldots, N_{l}
$$

By substituting the constitutive relations Eq. (5), the geometric relations Eq. (4) and the postulated, unified displacement field $\mathbf{u}=F_{\tau} \mathbf{u}_{\tau}$ into Eq. (22) and after some algebraic manipulations and application of Gauss-Green's theorem, DP statement reads:

$$
\begin{gathered}
\int_{\Omega_{k}} \delta \mathbf{u}_{\tau}^{k T} \int_{h_{k}}\left\{-F_{\tau} \mathbf{D}_{p}^{T}\left[\widetilde{\mathbf{C}}_{p p}^{k} F_{s} \mathbf{D}_{p}+\widetilde{\mathbf{C}}_{p n}^{k}\left(F_{s} \mathbf{D}_{n \Omega}+F_{s, z}\right)\right]+\left(-F_{\tau} \mathbf{D}_{n \Omega}^{T}+F_{\tau, z}\right)\right. \\
\left.\times\left[\widetilde{\mathbf{C}}_{n p}^{k} F_{s} \mathbf{D}_{p}+\widetilde{\mathbf{C}}_{n n}^{k}\left(F_{s} \mathbf{D}_{n \Omega}+F_{s, z}\right)\right]\right\} d z_{k} \mathbf{u}_{s}^{k} d \Omega_{k}+\int \Gamma_{k} \delta \mathbf{u}_{\tau}^{k T} \int_{h_{k}}\left\{F _ { \tau } \mathbf { I } _ { p } ^ { T } \left[\widetilde{\mathbf{C}}_{p p}^{k} F_{s} \mathbf{D}_{p}\right.\right. \\
\left.\left.+\widetilde{\mathbf{C}}_{p n}^{k}\left(F_{s} \mathbf{D}_{n \Omega}+F_{s, z}\right)\right]+F_{\tau} \mathbf{I}_{n \Omega}^{T}\left[\widetilde{\mathbf{C}}_{n p}^{k} F_{s} \mathbf{D}_{p}+\widetilde{\mathbf{C}}_{n n}^{k}\left(F_{s} \mathbf{D}_{n \Omega}+F_{s, z}\right)\right]\right\} d z_{k} \mathbf{u}_{s}^{k} d \Omega_{k}= \\
\int_{\Omega_{k}} \delta \mathbf{u}_{\tau}^{k T} \int_{h_{k}} \rho^{k} F_{\tau} F_{s} d z_{k} \mathbf{u}_{s}^{k} d \Omega_{k} k=1,2, \ldots, N_{l}
\end{gathered}
$$


Table 1

Assessment of CUF models: first dimensionless deterministic fundamental frequency $\bar{\omega}$ for $a_{0} / h_{0}=5$ and antisymmetric lamination

\begin{tabular}{lcccc}
\hline$E_{L 0} / E_{T 0}$ & 3 & 10 & 20 & 40 \\
\hline FSDT & 2.6413 & 3.3717 & 4.0128 & 4.7316 \\
ED2 & 2.6405 & 3.3502 & 3.9516 & 4.6028 \\
EDZ2 & 2.6378 & 3.3404 & 3.9322 & 4.5708 \\
Noor [27] & 2.6182 & 3.2578 & 3.7622 & 4.2719 \\
ED4 & 2.6032 & 3.2412 & 3.7465 & 4.2592 \\
LD2 & 2.6019 & 3.2377 & 3.7396 & 4.3352 \\
LM2 & 2.6019 & 3.2375 & 3.7389 & 4.2460 \\
LM4-LD4 & 2.6017 & 3.2371 & 3.7381 & 4.2444 \\
\hline
\end{tabular}

being:

$$
\mathbf{I}_{p}=\left[\begin{array}{lll}
1 & 0 & 0 \\
0 & 1 & 0 \\
1 & 1 & 0
\end{array}\right], \quad \mathbf{I}_{n \Omega}=\left[\begin{array}{lll}
0 & 0 & 1 \\
0 & 0 & 1 \\
0 & 0 & 0
\end{array}\right]
$$

Governing equations and boundary conditions are derived from Eq. (23):

$$
\begin{aligned}
& \mathbf{K}_{d}^{k \tau s} \mathbf{u}_{s}^{k}=\mathbf{M}_{d}^{k \tau s} \ddot{\mathbf{u}}_{s}^{k} \\
& \mathbf{u}_{\tau}^{k}=\overline{\mathbf{u}}_{\tau}^{k} \\
& \boldsymbol{\Pi}_{d}^{k \tau s} \mathbf{u}_{s}^{k}=\boldsymbol{\Pi}_{d}^{k \tau s} \overline{\mathbf{u}}_{s}^{k} \quad(x, y, z) \in \Gamma_{k}^{g} \\
& (x, y, z) \in \Gamma_{k}^{m}
\end{aligned}
$$

$\overline{\mathbf{u}}_{\tau}^{k}$ and $\Pi_{d}^{k \tau s} \overline{\mathbf{u}}_{s}^{k}$ are the $k$-layer geometrical and mechanical boundary conditions defined on $\Gamma_{k}^{g}$ and $\Gamma_{k}^{m}$, repsectively and:

$$
\begin{aligned}
& \mathbf{K}_{d}^{k \tau s}=\int_{h_{k}}\left\{-F_{\tau} \mathbf{D}_{p}^{T}\left[\widetilde{\mathbf{C}}_{p p}^{k} F_{s} \mathbf{D}_{p}+\widetilde{\mathbf{C}}_{p n}^{k}\left(F_{s} \mathbf{D}_{n \Omega}+F_{s, z}\right)\right]+\left(-F_{\tau} \mathbf{D}_{n \Omega}^{T}+F_{\tau, z}\right)\right. \\
& \left.\times\left[\widetilde{\mathbf{C}}_{n p}^{k} F_{s} \mathbf{D}_{p}+\widetilde{\mathbf{C}}_{n n}^{k}\left(F_{s} \mathbf{D}_{n \Omega}+F_{s, z}\right)\right]\right\} d z_{k} \\
& \boldsymbol{\Pi}_{d}^{k \tau s}=\int_{h_{k}}\left\{F_{\tau} \mathbf{I}_{p}^{T}\left[\widetilde{\mathbf{C}}_{p p}^{k} F_{s} \mathbf{D}_{p}+\widetilde{\mathbf{C}}_{p n}^{k}\left(F_{s} \mathbf{D}_{n \Omega}+F_{s, z}\right)\right]+F_{\tau} \mathbf{I}_{n \Omega}^{T}\left[\widetilde{\mathbf{C}}_{n p}^{k} F_{s} \mathbf{D}_{p}\right.\right. \\
& \left.\left.+\widetilde{\mathbf{C}}_{n n}^{k}\left(F_{s} \mathbf{D}_{n \Omega}+F_{s, z}\right)\right]\right\} d z_{k} \\
& \mathbf{M}_{d}^{k \tau s}=\int_{h_{k}} \rho^{k} F_{\tau} F_{s} d z_{k} \mathbf{I}
\end{aligned}
$$

being I the identity matrix. The displacement field presented in Eq. (11) and characterised for a $k$-layer represents the solution to the problem in Eq. (25).

\subsection{CUF models assessment}

CUF models are assessed toward the solution by Noor [27] in which the three-dimensional equilibrium equations were solved via a higher-order, mixed, finite difference scheme. The deterministic first fundamental frequency is investigated. It is put into a non-dimensionalised form according to the following formula:

$$
\bar{\omega}=10 \omega_{11} h_{0}^{2} \sqrt{\frac{\rho}{E_{T 0}}}
$$

A [0/90/0/90] square plate is considered. Stacking sequence starts from plate top. Plies orientation is measured toward $x$ coordinate. Layers have all the same thickness and they are all made of the same material. Material 
properties are: $G_{L T 0}=0.6 E_{T 0}, G_{T T 0}=0.5 E_{T 0}$ and $\nu_{L T 0}=\nu_{T T 0}=0.25$. Subscript 'L' represents a direction parallel to the fibres, while ' $T$ ' stands for a direction perpendicular to them. The ratio between $E_{L 0}$ and $E_{T 0}$ is considered as analysis parameter. It is as low as three and as high as 40 . The ratio between the reference side length and the reference global thickness $\left(a_{0} / h_{0}\right)$ is considered to be equal to five. A thick plate is, therefore, investigated. Results are presented in Table 1. ED4 theory and LW models are similar to the reference solution. Lower order models and FSDT are accurate for $E_{L 0} / E_{T 0}$ equal to three. LD2 and LM2 yield similar results for $E_{L 0} / E_{T 0}$ as high as ten. LW models converge to the same results.

\section{The Monte Carlo Method}

The Monte Carlo Method (MCM) is a procedure that allows to solve numerically those stochastic and deterministic problems for which analytical or approximated solution can not be profitably applied. It is especially useful in studying systems characterised by a large number of degrees of freedom or in which uncertainty plays a relevant role. Historical notes on MCM can be found in Metropolis [22] and Anderson [1]. A brief description of the method follows. Its detailed discussion can be found in Metropolis and Ulam [23], Kalos and Whitlock [15] and Rubinstein [36].

Being $\mathbf{X} \in \mathbb{R}^{N_{\text {inp }}}$ and $\Omega^{*} \in \mathbb{R}^{N_{\text {out }}}$ the vectors of input and output stochastic parameters, MCM consists of the generation of $N_{t o t}$ samples of $\Omega^{*}$ by means of the solution of $N_{t o t}$ deterministic problems (or simulations) for which the value of the input stochastic parameters has been randomly extracted according to appropriate Probability Density Functions (PDFs):

$$
\mathbf{X}_{i}=\left\{x_{1}, x_{2}, \ldots, x_{N_{\text {inp }}}\right\}_{i} \mapsto \Omega_{i}^{*}=\left\{\omega_{1}^{*}, \omega_{2}^{*}, \ldots, \omega_{N_{\text {out }}}^{*}\right\}_{i} i=1,2, \ldots, N_{\text {tot }}
$$

being $x_{j i}^{*}$ and $\omega_{j i}^{*}$ the $i^{t h}$ realisation of the $j^{t h}$ component of $\mathbf{X}$ and $\Omega^{*}$, respectively. Once a population of the stochastic variable $\Omega^{*}$ has been determined, the statistical moments (such as mean value and standard deviation) can be computed:

$$
\begin{aligned}
\mu_{\Omega^{*} j} & =\frac{1}{N_{t o t}} \sum_{i=i}^{N_{\text {tot }}} \omega_{i j}^{*}\left(\mathbf{x}_{i}\right) \\
\sigma_{\Omega^{*} j} & =\sqrt{\frac{1}{N_{\text {tot }}-1} \sum_{i=i}^{N_{\text {tot }}}\left[\omega_{i j}^{* 2}\left(\mathbf{x}_{i}\right)-\mu_{\Omega^{*} j}^{2}\right]} j=1,2, \ldots, N_{\text {out }}
\end{aligned}
$$

MCM is known to be very demanding in terms of computational effort and time. But it is, also, an accurate method that does not suffer the number of input and output stochastic variables, except that the computational effort is further increased. The computational time can be tackled adopting parallelised software and hardware architectures. Its efficiency can be increased, for instance, via variance reduction procedures (see Pradlwarterand and Schuëller [30] and Proppe et al. [31]). In this work, the computational cost does not represent a relevant problem since a closed form analytical solution is adopted. Several versions of the method can be identified on the basis of the sampling technique used for drawing the input stochastic parameters. Latin Hypercube Sampling Technique (LHST) (see McKay et al. [20]) is adopted here. According to LHST, the range of probable values for each uncertain input parameter $X_{j}$ is divided into $N_{t o t}$ ordered segments of equal probability $1 / N_{t o t}$. The whole sample space is partitioned into $N_{t o t}^{N_{i n p}}$ cells having equal probability. Each parameter is sampled once from each segment. LHST, therefore, yields an accurate description of tails of inputs' PDF and, consequently, a detailed insight into the extremes of outputs' PDF. Uniform and Weibull PDFs are sampled by means of the inversion method while Box-Muller's method is adopted to draw numbers according to a Gaussian PDF, see Fishman [10].

\section{Numerical results and discussion}

The effect of uncertainty in material properties and geometrical parameters on the first fundamental frequency is investigated. Two stacking sequences are considered: a [0/90/0] symmetric lamination and a [0/90/0/90] antisymmetric one. Two different orthotropic materials are adopted. Plates are considered to be nominally square. Deterministic results are firstly presented to better outline, through comparison, the effect of uncertainties. 
Table 2

Deterministic dimensionless fundamental frequency $\omega^{*}$ in the case of $a_{0} / h_{0}=100$ for both laminations and materials

\begin{tabular}{lccccc}
\hline & \multicolumn{2}{c}{$[0 / 90 / 0]$} & & \multicolumn{2}{c}{$[0 / 90 / 0 / 90]$} \\
\cline { 2 - 3 } \cline { 6 - 6 } & Mat I & Mat II & & Mat I & Mat II \\
\hline FSDT & 18.845 & 15.191 & & 17.284 & 14.029 \\
ED2 & 18.845 & 15.191 & & 17.282 & 14.029 \\
EDZ2 & 18.838 & 15.169 & & 17.281 & 14.023 \\
LD2 & 18.835 & 15.168 & & 17.273 & 14.013 \\
LD4 & 18.835 & 15.168 & & 17.273 & 14.013 \\
LM2 & 18.835 & 15.168 & & 17.273 & 14.013 \\
LM4 & 18.835 & 15.168 & & 17.273 & 14.013 \\
\hline
\end{tabular}

Table 3

Deterministic dimensionless fundamental frequency $\omega^{*}$ in the case of $a_{0} / h_{0}=10$ for both laminations and materials

\begin{tabular}{lccccc}
\hline & \multicolumn{2}{c}{$[0 / 90 / 0]$} & & \multicolumn{2}{c}{$[0 / 90 / 0 / 90]$} \\
\cline { 2 - 3 } \cline { 6 - 6 } & Mat I & Mat II & & Mat I & Mat II \\
\hline FSDT & 15.583 & 12.581 & & 15.233 & 12.320 \\
ED2 & 15.582 & 12.577 & & 15.055 & 12.281 \\
EDZ2 & 15.216 & 11.661 & & 15.003 & 11.952 \\
LD2 & 15.097 & 11.604 & & 14.498 & 11.384 \\
LD4 & 15.069 & 11.585 & & 14.495 & 11.382 \\
LM2 & 15.082 & 11.591 & & 14.497 & 11.383 \\
LM4 & 15.069 & 11.585 & & 14.495 & 11.382 \\
\hline
\end{tabular}

\subsection{Deterministic natural frequencies}

Reference deterministic properties for the first material are the same as those assumed in subsection 2.6 with $E_{L 0}=40 E_{T 0}$. In the case of the second material: $E_{L 0}=25 E_{T 0}, G_{L T 0}=0.5 E_{T 0}, G_{T T 0}=0.2 E_{T 0}$ and $\nu_{L T 0}=\nu_{T T 0}=0.25$. For the first material, the anisotropy is mainly pertinent to the Young moduli. For the second one, the difference between the shear moduli is also remarkable. The density is the same for both the materials. Plate sides length is $a_{0}=b_{0}=0.1 \mathrm{~m}$. The side-to-thickness ratio, $a_{0} / h_{0}$, is considered to be equal to 100 and ten. Thin and relatively thick plates are, therefore, investigated. In the case of the symmetric laminate, the middle layer thickness is twice the one of the external layers. For the anti-symmetric plate, all the plies have the same thickness. The deterministic natural frequency is put into a non-dimensionalised form in the following manner:

$$
\omega^{*}=\omega_{11} \frac{a_{0}^{2}}{h_{0}} \sqrt{\frac{\rho}{E_{T 0}}}
$$

Deterministic dimensionless fundamental natural frequency $\omega^{*}$ is presented in Tables 2 and 3 . Since the second material is less stiff than the first one and being the density the same, the frequency for the second material is expected to be lower than for the first one. As far as a hierarchical classification of CUF models is concerned, the more accurate the model, the lower the value of the frequency. This is intuitively due to the fact that the more accurate the theory, the higher the number of degrees of freedom. Increasing the polynomial approximation order, LW models based on DP and RMVT yield the same results. Being fixed the side-to-thickness ratio and the material type, the anti-symmetric plate is less stiff than the symmetric one. In the case of thin plates, FSDT and higher order models yield matching results. For $a_{0} / h_{0}=10$, results via FSDT can differ by about $8 \%$ from those obtained by LM4 and LD4 models. For a fixed stacking sequence and side-to-thickness ratio, the difference between results obtained via FSDT and higher order models is higher in the case of the second material since it presents a higher degree of anisotropy than the first one.

\subsection{Stochastic natural frequencies}

As far as a stochastic approach is concerned, several statistical tests are carried out considering different PDFs for the input stochastic variables. Mean values are coincident to the deterministic reference values. 
Geometrical parameters (plate's sides length and plies' thickness) are distributed according to: 1- a uniform PDF with a percentage variation with respect to the mean value equal to \pm 5 , that is, the coefficient of variation $(\mathrm{CV} \%)$ is equal to 2.88 or 2- a Gaussian PDF with CV\% equal to five. The first case is addressed as 'GU', the second one as 'GG'. 'GD' stands for the case in which geometrical parameters are deterministic.

As far as material properties are concerned, a review of the ways to characterise and quantify uncertainty at both micro-mechanical and structural level was presented by Sriramula and Chryssanthopoulos [47]. Gaussian, log-normal and Weibull PDFs are widely used probability laws. As recommended in the Military Handbook MILHDBK-17-1F [29], two-parameters Weibull PDF should be preferred to Gaussian or log-normal models. In some cases, anyway, materials properties were found to be distributed according to a Gaussian PDF (see, for instance, Nakayasu and Maekawa [26] and Jeong and Shenoi [14]).

In this work, the elastic properties of each ply (Young's and shear moduli and Poisson's ratios) are drawn according to: 1- a two-parameters Weibull PDF (case EW) or 2- a Gaussian PDF (case EG). In both cases, the CV\% is equal to ten. 'ED' stands for deterministic elastic properties. Material density is sampled according to a two-parameters Weibull PDF (case DW) or a Gaussian PDF (case DG) or it can be deterministic (case DD). Although the Gaussian $\mathrm{PDF}$ is unbounded, the physics of the problem is preserved since, for instance, the probability that a material property is lower than or equal to zero is $10^{-24}$. In the case of geometrical parameters, this probability is even smaller.

It should underline that a limitation of the present study consists in the assumption of uncorrelated stochastic parameters. Sriramula and Chryssanthopoulos [46], for instance, showed that material strength can be significantly dependent on the plate thickness. Correlation among input stochastic variables will be investigated in a future study.

The dimensionless stochastic fundamental natural frequency is:

$$
\Omega^{*}=\Omega_{11} \frac{a_{0}^{2}}{h_{0}} \sqrt{\frac{\rho}{E_{T 0}}}
$$

It is statistically described in terms of mean value $\left(\mu_{\Omega^{*}}\right)$, standard deviation $\left(\sigma_{\Omega^{*}}\right)$, percentage coefficient of variation $\left(\mathrm{CV}_{\Omega^{*}} \%\right)$ and Cumulative Distribution Function. Confidence intervals of mean value $\left(\mathrm{CI}_{0.95}^{\mu_{\Omega^{*}}}\right)$ and standard deviation $\left(\mathrm{CI}_{0.95}^{\sigma_{\Omega^{*}}}\right)$ are also computed. A confidence level equal to 0.95 is considered. The total number of simulations $\left(N_{t o t}\right)$ is $3 \cdot 10^{4}$. A mode tracking (see Blelloch [3]) is not required since the eigenmode with $m=n=1$ in Eq. (11) is considered.

The statistic estimators of $\Omega^{*}$ for thin and relatively thick symmetric laminates made of the first material are presented in Tables 4 and 5, respectively. The effect of uncertainty is investigated considering: random geometrical parameters only (test 1: GU, ED, DD), random material elastic properties only (test 2: GD, EW, DD) and all input parameters to be random (test 3: GU, EW, DW). The stochastic perturbation of the input stochastic parameters is symmetric with respect to the reference values. Difference between the deterministic natural frequency and its mean value is due to a non-linear dependency from the input parameters. For the tests 1 and 3 , there is a probability of 0.95 , at least, that $\mu_{\Omega^{*}}$ is higher than $\omega^{*}$. In the test $2, \mu_{\Omega^{*}}$ is lower than $\omega^{*}$ with the same probability. Uncertainty in geometrical parameters is more relevant than randomness in the material properties. In the test $1, \mathrm{CV}_{\Omega^{*}} \%$ is higher than the CV\% of the geometrical parameters. As shown in test 2, the fundamental natural frequency is not very sensitive to the uncertainty in the material elastic properties. As far as CUF models are concerned, all the considered theories yield statistically matching results. This is, also, shown in Fig. 6 in which the CDF of $\Omega^{*}$, normalised versus the mean value, is presented. The CDF has been computed via all the considered CUF models in the case of test 3 for both thin and relatively thick plates. Fig. 6 also shows that a correlation between the thickness of the plate and $\Omega^{*}$ is present: the thicker the plate, the smaller the coefficient of variation. In the case of $a_{0} / h_{0}=10$, the difference in the mean value is due to the difference in the number of degrees of freedom as observed in the deterministic analyses. Tests 1 to 3 have been carried out also in the case of thin and thick plates made of the second material. Results are presented in Tables 6 and 7. CDF for all the tests and $a_{0} / h_{0}=10$ is reported in Fig. 7. The same consideration addressed for the first material are valid. Considering uncertainty affecting one input parameter at time, the mean value does not depend on the shear moduli in the case of thin plates. In the case of $a_{0} / h_{0}=10$, the shear deformation becomes important. FSDT yields a mean value sensitive to $G_{L T}$ only, while it depends also on $G_{T T}$ in the case of higher order models.

In the case of anti-symmetric laminates made of the first material, elastic parameters are considered to be random according to a Weibull PDF (test 2) and a Gaussian one (test 2a). Tables 8 and 9 present the results for thin and 
Table 4

$\Omega^{*}$ statistic estimators in the case of the symmetric laminate, first material and $a_{0} / h_{0}=100$

\begin{tabular}{|c|c|c|c|c|c|}
\hline & $\mu_{\Omega^{*}}$ & $\mathrm{CI}_{0.95}^{\mu_{\Omega^{*}}}$ & $10 \times \sigma_{\Omega^{*}}$ & $10 \times \mathrm{CI}_{0.95}^{\sigma \Omega^{*}}$ & $\mathrm{CV}_{\Omega^{*}} \%$ \\
\hline \multicolumn{6}{|c|}{ Test 1: GU, ED, DD } \\
\hline FSDT & 18.896 & $(18.885,18.907)$ & 9.844 & $(9.766,9.924)$ & 5.21 \\
\hline ED2 & 18.896 & $(18.885,18.907)$ & 9.810 & $(9.732,9.889)$ & 5.19 \\
\hline EDZ2 & 18.889 & $(18.878,18.900)$ & 9.780 & $(9.703,9.859)$ & 5.18 \\
\hline LD2 & 18.886 & $(18.875,18.898)$ & 9.811 & $(9.733,9.890)$ & 5.19 \\
\hline LD4 & 18.886 & $(18.875,18.897)$ & 9.813 & $(9.736,9.893)$ & 5.20 \\
\hline LM2 & 18.886 & $(18.875,18.897)$ & 9.816 & $(9.738,9.895)$ & 5.20 \\
\hline LM4 & 18.886 & $(18.875,18.897)$ & 9.833 & $(9.755,9.913)$ & 5.21 \\
\hline \multicolumn{6}{|c|}{ Test 2: GD, EW, DD } \\
\hline FSDT & 18.801 & $(18.794,18.807)$ & 5.710 & $(5.665,5.756)$ & 3.04 \\
\hline ED2 & 18.801 & $(18.794,18.807)$ & 5.698 & $(5.653,5.744)$ & 3.03 \\
\hline EDZ2 & 18.793 & $(18.787,18.800)$ & 5.697 & $(5.652,5.743)$ & 3.03 \\
\hline LD2 & 18.791 & $(18.784,18.797)$ & 5.704 & $(5.659,5.750)$ & 3.04 \\
\hline LD4 & 18.790 & $(18.784,18.797)$ & 5.715 & $(5.669,5.761)$ & 3.04 \\
\hline LM2 & 18.790 & $(18.784,18.797)$ & 5.683 & $(5.638,5.729)$ & 3.02 \\
\hline LM4 & 18.790 & $(18.784,18.797)$ & 5.683 & $(5.638,5.729)$ & 3.02 \\
\hline \multicolumn{6}{|c|}{ Test 3: GU, EW, DW } \\
\hline FSDT & 18.879 & $(18.864,18.893)$ & 12.79 & $(12.69,12.89)$ & 6.78 \\
\hline ED2 & 18.879 & $(18.865,18.894)$ & 12.86 & $(12.76,12.97)$ & 6.81 \\
\hline EDZ2 & 18.871 & $(18.857,18.886)$ & 12.79 & $(12.69,12.89)$ & 6.78 \\
\hline LD2 & 18.869 & $(18.855,18.884)$ & 12.88 & $(12.78,12.98)$ & 6.83 \\
\hline LD4 & 18.869 & $(18.854,18.883)$ & 12.82 & $(12.71,12.92)$ & 6.79 \\
\hline LM2 & 18.869 & $(18.855,18.884)$ & 12.87 & $(12.77,12.98)$ & 6.82 \\
\hline LM4 & 18.869 & $(18.854,18.883)$ & 12.83 & $(12.73,12.93)$ & 6.80 \\
\hline
\end{tabular}

Table 5

$\Omega^{*}$ statistic estimators in the case of the symmetric laminate, first material and $a_{0} / h_{0}=10$

\begin{tabular}{lccccc}
\hline \multicolumn{7}{c}{$\mu_{\Omega^{*}}$} & $\mathrm{CI}_{0.95}^{\mu_{\Omega^{*}}}$ & $10 \times \sigma_{\Omega^{*}}$ & $10 \times \mathrm{CI}_{0.95}^{\sigma} \Omega^{*}$ & $\mathrm{CV}_{\Omega^{*}} \%$ \\
\hline FSDT & 15.614 & $(15.607,15.621)$ & 6.443 & $(6.3924,6.495)$ & 4.13 \\
ED2 & 15.613 & $(15.606,15.620)$ & 6.448 & $(6.3971,6.500)$ & 4.13 \\
EDZ2 & 15.245 & $(15.238,15.252)$ & 6.145 & $(6.0962,6.195)$ & 4.03 \\
LD2 & 15.125 & $(15.119,15.132)$ & 6.093 & $(6.0445,6.142)$ & 4.03 \\
LD4 & 15.097 & $(15.090,15.104)$ & 6.065 & $(6.0176,6.114)$ & 4.02 \\
LM2 & 15.110 & $(15.103,15.117)$ & 6.037 & $(5.9894,6.086)$ & 4.00 \\
LM4 & 15.097 & $(15.090,15.104)$ & 6.058 & $(6.0102,6.107)$ & 4.01 \\
& & \multicolumn{2}{c}{ Test 2: GD, EW, DD } & & \\
FSDT & 15.548 & $(15.544,15.552)$ & 3.401 & $(3.374,3.428)$ & 2.19 \\
ED2 & 15.542 & $(15.538,15.545)$ & 3.411 & $(3.384,3.438)$ & 2.19 \\
EDZ2 & 15.167 & $(15.163,15.171)$ & 3.512 & $(3.484,3.541)$ & 2.32 \\
LD2 & 15.048 & $(15.044,15.052)$ & 3.433 & $(3.406,3.461)$ & 2.28 \\
LD4 & 15.019 & $(15.015,15.023)$ & 3.422 & $(3.395,3.450)$ & 2.28 \\
LM2 & 15.032 & $(15.029,15.036)$ & 3.426 & $(3.399,3.454)$ & 2.28 \\
LM4 & 15.019 & $(15.015,15.023)$ & 3.440 & $(3.413,3.468)$ & 2.29 \\
& & \multicolumn{5}{c}{ Test 3: GU, EW, DW } & & \\
FSDT & 15.601 & $(15.591,15.611)$ & 8.778 & $(8.708,8.848)$ & 5.63 \\
ED2 & 15.594 & $(15.584,15.604)$ & 8.703 & $(8.634,8.774)$ & 5.58 \\
EDZ2 & 15.218 & $(15.208,15.228)$ & 8.575 & $(8.507,8.644)$ & 5.63 \\
LD2 & 15.098 & $(15.089,15.108)$ & 8.479 & $(8.411,8.547)$ & 5.62 \\
LD4 & 15.069 & $(15.060,15.079)$ & 8.443 & $(8.376,8.511)$ & 5.60 \\
LM2 & 15.082 & $(15.073,15.092)$ & 8.442 & $(8.375,8.510)$ & 5.60 \\
LM4 & 15.069 & $(15.060,15.079)$ & 8.488 & $(8.421,8.557)$ & 5.63 \\
\hline
\end{tabular}


Table 6

$\Omega^{*}$ statistic estimators in the case of the symmetric laminate, second material and $a_{0} / h_{0}=100$.

\begin{tabular}{|c|c|c|c|c|c|}
\hline & $\mu_{\Omega^{*}}$ & $\mathrm{CI}_{0.95}^{\mu_{\Omega^{*}}}$ & $10 \times \sigma_{\Omega^{*}}$ & $10 \times \mathrm{CI}_{0.95}^{\sigma_{\Omega^{*}}}$ & $\mathrm{CV}_{\Omega *} \%$ \\
\hline \multicolumn{6}{|c|}{ Test 1: GU, ED, DD } \\
\hline FSDT & 15.232 & $(15.224,15.241)$ & 7.831 & $(7.769,7.894)$ & 5.14 \\
\hline ED2 & 15.232 & $(15.224,15.241)$ & 7.826 & $(7.764,7.889)$ & 5.14 \\
\hline EDZ2 & 15.211 & $(15.202,15.219)$ & 7.794 & $(7.732,7.857)$ & 5.12 \\
\hline LD2 & 15.209 & $(15.200,15.218)$ & 7.792 & $(7.730,7.855)$ & 5.12 \\
\hline LD4 & 15.209 & $(15.200,15.218)$ & 7.780 & $(7.719,7.843)$ & 5.12 \\
\hline LM2 & 15.209 & $(15.200,15.218)$ & 7.795 & $(7.733,7.858)$ & 5.12 \\
\hline LM4 & 15.209 & $(15.200,15.218)$ & 7.796 & $(7.735,7.859)$ & 5.13 \\
\hline \multicolumn{6}{|c|}{ Test 2: GD, EW, DD } \\
\hline FSDT & 15.157 & $(15.152,15.162)$ & 4.426 & $(4.391,4.462)$ & 2.92 \\
\hline ED2 & 15.157 & $(15.152,15.162)$ & 4.419 & $(4.384,4.455)$ & 2.91 \\
\hline EDZ2 & 15.135 & $(15.130,15.140)$ & 4.380 & $(4.345,4.415)$ & 2.89 \\
\hline LD2 & 15.134 & $(15.129,15.139)$ & 4.399 & $(4.364,4.435)$ & 2.91 \\
\hline LD4 & 15.134 & $(15.129,15.139)$ & 4.397 & $(4.362,4.432)$ & 2.90 \\
\hline LM2 & 15.134 & $(15.129,15.139)$ & 4.405 & $(4.370,4.441)$ & 2.91 \\
\hline LM4 & 15.133 & $(15.128,15.138)$ & 4.389 & $(4.354,4.424)$ & 2.90 \\
\hline \multicolumn{6}{|c|}{ Test 3: GU, EW, DW } \\
\hline FSDT & 15.221 & $(15.210,15.233)$ & 10.19 & $(10.11,10.27)$ & 6.69 \\
\hline ED2 & 15.221 & $(15.210,15.233)$ & 10.26 & $(10.17,10.34)$ & 6.74 \\
\hline EDZ2 & 15.199 & $(15.187,15.210)$ & 10.15 & $(10.07,10.22)$ & 6.68 \\
\hline LD2 & 15.196 & $(15.185,15.208)$ & 10.10 & $(10.02,10.18)$ & 6.64 \\
\hline LD4 & 15.196 & $(15.185,15.208)$ & 10.16 & $(10.08,10.24)$ & 6.68 \\
\hline LM2 & 15.196 & $(15.185,15.208)$ & 10.11 & $(10.03,10.19)$ & 6.65 \\
\hline LM4 & 15.196 & $(15.185,15.208)$ & 10.17 & $(10.09,10.26)$ & 6.70 \\
\hline
\end{tabular}

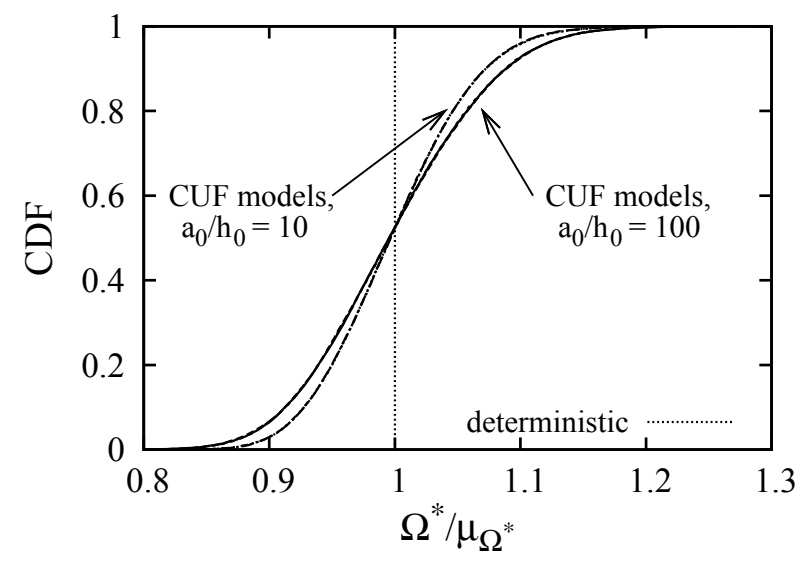

Fig. 6. Cumulative Distribution Function for the symmetric laminate, first material, $a_{0} / h_{0}=100$ and $a_{0} / h_{0}=10$, test 3 .

relatively thick plates. The tests yield similar statistic results. $\Omega^{*}$ is not very sensitive to stochastic perturbations in the material elastic properties. Both Weibull or Gaussian PDFs can be assumed as probabilistic model for the elastic properties in order to evaluate the second order statistics of $\Omega^{*}$. The difference in the standard deviations is mainly due to the difference in the left tail of the CDFs as shown in Fig. 8. For an anti-symmetric laminate made of the second material, tests have been done considering all the input parameters to be random. In test 4 (GG, EW, DW) a Weibull PDF is assumed as probabilistic model for material properties, while in test 5 (GG, EG, DG) a Gaussian PDF is used. Geometrical data are sampled according to a Gaussian PDF. Results are presented in Tables 10 and 11. Since results are similar, both a Weibull or a Gaussian PDFs can be also assumed for the material density, unless small fractile values are requieed. The high value of $\mathrm{CV}_{\Omega^{*}} \%$ is due to the probabilistic model assumed for the geometrical parameters. 
Table 7

$\Omega^{*}$ statistic estimators in the case of the symmetric laminate, second material and $a_{0} / h_{0}=10$

\begin{tabular}{|c|c|c|c|c|c|}
\hline & $\mu_{\Omega^{*}}$ & $\mathrm{CI}_{0.95}^{\mu_{\Omega^{*}}}$ & $10 \times \sigma_{\Omega^{*}}$ & $10 \times \mathrm{CI}_{095}^{\sigma_{\Omega^{*}}}$ & $\mathrm{CV}_{\Omega^{*}} \%$ \\
\hline \multicolumn{6}{|c|}{ Test 1: GU, ED, DD } \\
\hline FSDT & 12.606 & $(12.600,12.612)$ & 5.135 & $(5.095,5.177)$ & 4.07 \\
\hline ED2 & 12.602 & $(12.596,12.608)$ & 5.140 & $(5.100,5.182)$ & 4.08 \\
\hline EDZ2 & 11.684 & $(11.679,11.689)$ & 4.389 & $(4.354,4.425)$ & 3.76 \\
\hline LD2 & 11.626 & $(11.621,11.631)$ & 4.377 & $(4.343,4.413)$ & 3.76 \\
\hline LD4 & 11.607 & $(11.602,11.612)$ & 4.353 & $(4.318,4.388)$ & 3.75 \\
\hline LM2 & 11.614 & $(11.609,11.619)$ & 4.369 & $(4.334,4.404)$ & 3.76 \\
\hline LM4 & 11.607 & $(11.602,11.612)$ & 4.355 & $(4.320,4.390)$ & 3.75 \\
\hline \multicolumn{6}{|c|}{ Test 2: GD, EW, DD } \\
\hline FSDT & 12.555 & $(12.552,12.558)$ & 2.670 & $(2.648,2.691)$ & 2.13 \\
\hline ED2 & 12.545 & $(12.542,12.548)$ & 2.685 & $(2.664,2.707)$ & 2.14 \\
\hline EDZ2 & 11.628 & $(11.625,11.631)$ & 2.684 & $(2.662,2.705)$ & 2.31 \\
\hline LD2 & 11.571 & $(11.568,11.574)$ & 2.645 & $(2.624,2.667)$ & 2.29 \\
\hline LD4 & 11.552 & $(11.549,11.555)$ & 2.636 & $(2.615,2.657)$ & 2.28 \\
\hline LM2 & 11.558 & $(11.555,11.561)$ & 2.633 & $(2.612,2.654)$ & 2.28 \\
\hline LM4 & 11.552 & $(11.549,11.555)$ & 2.641 & $(2.620,2.663)$ & 2.29 \\
\hline \multicolumn{6}{|c|}{ Test 3: GU, EW, DW } \\
\hline FSDT & 12.597 & $(12.589,12.605)$ & 7.001 & $(6.945,7.057)$ & 5.56 \\
\hline ED2 & 12.588 & $(12.580,12.595)$ & 6.996 & $(6.940,7.052)$ & 5.56 \\
\hline EDZ2 & 11.667 & $(11.660,11.674)$ & 6.305 & $(6.255,6.356)$ & 5.40 \\
\hline LD2 & 11.610 & $(11.603,11.617)$ & 6.309 & $(6.259,6.360)$ & 5.43 \\
\hline LD4 & 11.590 & $(11.583,11.598)$ & 6.284 & $(6.234,6.334)$ & 5.42 \\
\hline LM2 & 11.597 & $(11.590,11.604)$ & 6.289 & $(6.239,6.339)$ & 5.42 \\
\hline LM4 & 11.591 & $(11.584,11.598)$ & 6.298 & $(6.248,6.348)$ & 5.43 \\
\hline
\end{tabular}

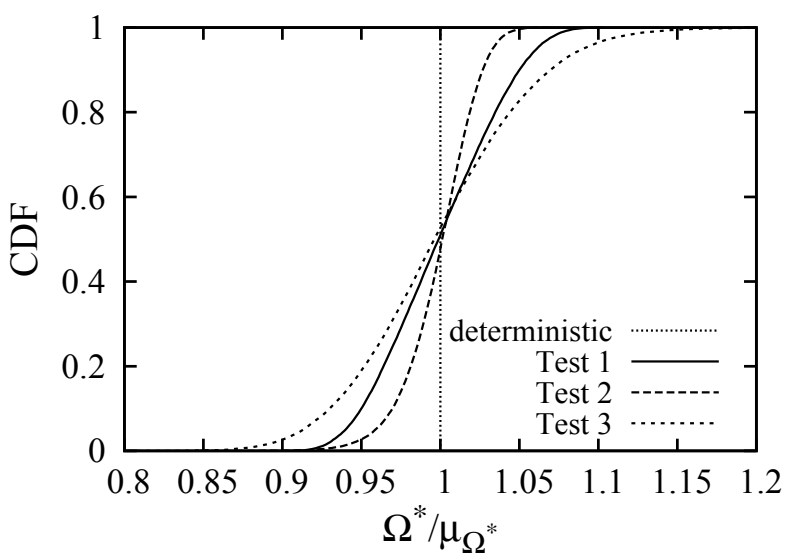

Fig. 7. Cumulative Distribution Function for the symmetric laminate, second material, $a_{0} / h_{0}=10$, tests 1,2 , and 3 .

\section{Concluding remarks}

The statistic determination of the fundamental natural frequency, $\Omega^{*}$, of the first bending eigenmode has been carried out through the Monte Carlo Method. Simply supported, symmetric and anti-symmetric cross-ply plates have been analysed. Thin and relatively thick laminates have been considered: the side-to-thickness parameter, $a_{0} / h_{0}$, equals 100 and 10. The effect of the anisotropy due to the difference between the Young's moduli and between the shear moduli has been investigated. Both material properties and geometrical parameters have been considered to be random. Carrera's Unified Formulation (CUF) has been assumed to formulate classical and refined two-dimensional plate models. In the case of thin plates, all of the considered models yield similar results for both deterministic and stochastic approaches. For $a_{0}=h_{0}=10$, the hierarchy among the model becomes evident. In particular, in the case of the anti-symmetric stacking sequence and anisotropy affecting both Young's and shear moduli, classical 
Table 8

$\Omega^{*}$ statistic estimators in the case of the anti-symmetric laminate, first material and $a_{0} / h_{0}=100$.

\begin{tabular}{lccccc}
\hline \multicolumn{7}{c}{$\mu_{\Omega^{*}}$} & $\mathrm{CI}_{0.95}^{\mu_{\Omega^{*}}}$ & $10 \times \sigma_{\Omega^{*}}$ & $10 \times \mathrm{CI}_{0.95}^{\sigma}$ & $\mathrm{CV}_{\Omega^{*}} \%$ \\
\hline FSDT & 17.245 & $(17.240,17.249)$ & 4.026 & $(3.994,4.059)$ & 2.33 \\
ED2 & 17.243 & $(17.238,17.247)$ & 4.008 & $(3.976,4.040)$ & 2.32 \\
EDZ2 & 17.241 & $(17.237,17.246)$ & 4.010 & $(3.978,4.042)$ & 2.32 \\
LD2 & 17.233 & $(17.228,17.238)$ & 3.999 & $(3.967,4.031)$ & 2.32 \\
LD4 & 17.233 & $(17.228,17.237)$ & 3.996 & $(3.964,4.028)$ & 2.32 \\
LM2 & 17.233 & $(17.228,17.237)$ & 4.012 & $(3.980,4.045)$ & 2.33 \\
LM4 & 17.233 & $(17.228,17.237)$ & 4.008 & $(3.976,4.040)$ & 2.33 \\
& & \multicolumn{2}{c}{ Test 2a: GD, EG, DD } & & \\
FSDT & 17.247 & $(17.243,17.252)$ & 3.857 & $(3.826,3.888)$ & 2.24 \\
ED2 & 17.244 & $(17.239,17.248)$ & 3.850 & $(3.819,3.881)$ & 2.23 \\
EDZ2 & 17.243 & $(17.239,17.248)$ & 3.885 & $(3.854,3.916)$ & 2.25 \\
LD2 & 17.235 & $(17.231,17.239)$ & 3.853 & $(3.822,3.884)$ & 2.23 \\
LD4 & 17.234 & $(17.230,17.238)$ & 3.857 & $(3.826,3.888)$ & 2.24 \\
LM2 & 17.235 & $(17.230,17.239)$ & 3.853 & $(3.823,3.884)$ & 2.24 \\
LM4 & 17.233 & $(17.229,17.238)$ & 3.880 & $(3.849,3.911)$ & 2.25 \\
\hline
\end{tabular}

Table 9

$\Omega^{*}$ statistic estimators in the case of the anti-symmetric laminate, first material and $a_{0} / h_{0}=10$.

\begin{tabular}{lccccc}
\hline & $\mu_{\Omega^{*}}$ & $\mathrm{CI}_{0.95}^{\mu_{\Omega^{*}}}$ & $10 \times \sigma_{\Omega^{*}}$ & $10 \times \mathrm{CI}_{0.95}^{\sigma}$ & $\mathrm{CV}_{\Omega^{*}} \%$ \\
\hline FSDT & 15.197 & $(15.194,15.201)$ & 2.932 & $(2.909,2.956)$ & 1.93 \\
ED2 & 15.016 & $(15.013,15.019)$ & 2.865 & $(2.843,2.889)$ & 1.91 \\
EDZ2 & 14.960 & $(14.957,14.963)$ & 2.846 & $(2.823,2.869)$ & 1.90 \\
LD2 & 14.450 & $(14.447,14.453)$ & 2.642 & $(2.621,2.663)$ & 1.82 \\
LD4 & 14.446 & $(14.443,14.449)$ & 2.634 & $(2.613,2.655)$ & 1.82 \\
LM2 & 14.448 & $(14.445,14.451)$ & 2.641 & $(2.620,2.662)$ & 1.83 \\
LM4 & 14.446 & $(14.443,14.449)$ & 2.650 & $(2.629,2.671)$ & 1.83 \\
& & Test 2a: GD, EG, DD & & \\
FSDT & 15.198 & $(15.195,15.202)$ & 2.798 & $(2.776,2.820)$ & 1.84 \\
ED2 & 15.017 & $(15.014,15.020)$ & 2.748 & $(2.727,2.771)$ & 1.83 \\
EDZ2 & 14.962 & $(14.959,14.965)$ & 2.723 & $(2.702,2.745)$ & 1.82 \\
LD2 & 14.452 & $(14.449,14.455)$ & 2.509 & $(2.489,2.529)$ & 1.74 \\
LD4 & 14.449 & $(14.446,14.452)$ & 2.508 & $(2.489,2.529)$ & 1.74 \\
LM2 & 14.450 & $(14.447,14.452)$ & 2.494 & $(2.474,2.514)$ & 1.73 \\
LM4 & 14.448 & $(14.445,14.451)$ & 2.516 & $(2.496,2.536)$ & 1.74 \\
\hline
\end{tabular}

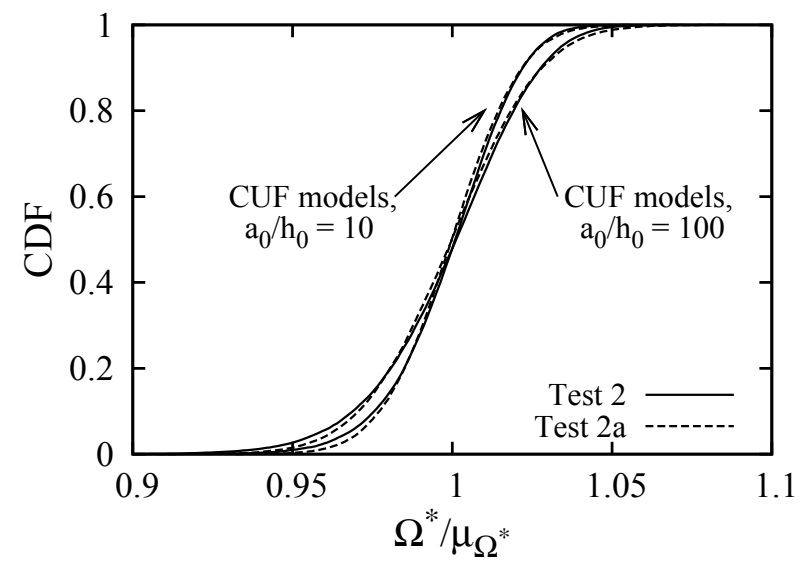

Fig. 8. Cumulative Distribution Function for the anti-symmetric laminate, first material, $a_{0} / h_{0}=100$ and $a_{0} / h_{0}=10$, tests 2 and $2 \mathrm{a}$. 
Table 10

$\Omega^{*}$ statistic estimators in the case of the anti-symmetric laminate, second material and $a_{0} / h_{0}=100$

\begin{tabular}{lccccc}
\hline \multicolumn{7}{c}{$\mu_{\Omega^{*}}$} & $\mathrm{CI}_{0.95}^{\mu_{\Omega^{*}}}$ & $\sigma_{\Omega^{*}}$ & $\mathrm{CI}_{0.95}^{\sigma} \Omega^{*}$ & $\mathrm{CV}_{\Omega^{*}} \%$ \\
\hline FSDT & 14.145 & $(14.132,14.159)$ & 1.190 & $(1.180,1.199)$ & 8.41 \\
ED2 & 14.144 & $(14.131,14.158)$ & 1.187 & $(1.178,1.197)$ & 8.39 \\
EDZ2 & 14.145 & $(14.131,14.158)$ & 1.183 & $(1.173,1.192)$ & 8.36 \\
LD2 & 14.129 & $(14.116,14.142)$ & 1.191 & $(1.182,1.201)$ & 8.43 \\
LD4 & 14.126 & $(14.113,14.140)$ & 1.183 & $(1.174,1.193)$ & 8.38 \\
LM2 & 14.133 & $(14.119,14.146)$ & 1.195 & $(1.186,1.205)$ & 8.46 \\
LM4 & 14.123 & $(14.110,14.137)$ & 1.183 & $(1.173,1.192)$ & 8.37 \\
& & Test 5: GG, EG, DG & & \\
FSDT & 14.142 & $(14.129,14.156)$ & 1.185 & $(1.175,1.194)$ & 8.38 \\
ED2 & 14.148 & $(14.134,14.161)$ & 1.184 & $(1.175,1.193)$ & 8.37 \\
EDZ2 & 14.139 & $(14.125,14.152)$ & 1.184 & $(1.175,1.194)$ & 8.37 \\
LD2 & 14.126 & $(14.113,14.139)$ & 1.179 & $(1.170,1.189)$ & 8.35 \\
LD4 & 14.119 & $(14.106,14.133)$ & 1.178 & $(1.169,1.188)$ & 8.34 \\
LM2 & 14.125 & $(14.111,14.138)$ & 1.181 & $(1.172,1.191)$ & 8.36 \\
LM4 & 14.130 & $(14.116,14.143)$ & 1.183 & $(1.174,1.193)$ & 8.37 \\
\hline
\end{tabular}

Table 11

$\Omega^{*}$ statistic estimators in the case of the anti-symmetric laminate, first material and $a_{0} / h_{0}=10$

\begin{tabular}{lccccc}
\hline \multicolumn{7}{c}{$\mu_{\Omega^{*}}$} & $\mathrm{CI}_{0.95}^{\mu_{\Omega^{*}}}$ & $10 \times \sigma_{\Omega^{*}}$ & $10 \times \mathrm{CI}_{0.95}^{\sigma \Omega^{*}}$ & $\mathrm{CV}_{\Omega^{*}} \%$ \\
\hline FSDT & 12.388 & $(12.377,12.398)$ & 9.166 & $(9.094,9.240)$ & 7.40 \\
ED2 & 12.348 & $(12.338,12.358)$ & 9.107 & $(9.035,9.180)$ & 7.37 \\
EDZ2 & 12.014 & $(12.004,12.023)$ & 8.679 & $(8.610,8.749)$ & 7.22 \\
LD2 & 11.432 & $(11.423,11.441)$ & 7.986 & $(7.923,8.051)$ & 6.99 \\
LD4 & 11.426 & $(11.417,11.435)$ & 7.951 & $(7.888,8.015)$ & 6.99 \\
LM2 & 11.433 & $(11.424,11.442)$ & 7.932 & $(7.869,7.996)$ & 6.94 \\
LM4 & 11.429 & $(11.420,11.438)$ & 7.943 & $(7.880,8.007)$ & 6.95 \\
& & Test 5: GG, EG, DG & & \\
FSDT & 12.391 & $(12.380,12.401)$ & 9.141 & $(9.069,9.215)$ & 7.38 \\
ED2 & 12.351 & $(12.341,12.362)$ & 9.100 & $(9.027,9.173)$ & 7.37 \\
EDZ2 & 12.015 & $(12.005,12.024)$ & 8.582 & $(8.513,8.651)$ & 7.14 \\
LD2 & 11.436 & $(11.427,11.445)$ & 7.886 & $(7.824,7.950)$ & 6.90 \\
LD4 & 11.430 & $(11.421,11.439)$ & 7.939 & $(7.876,8.003)$ & 6.95 \\
LM2 & 11.438 & $(11.429,11.447)$ & 7.947 & $(7.884,8.011)$ & 6.95 \\
LM4 & 11.430 & $(11.421,11.439)$ & 7.923 & $(7.860,7.987)$ & 6.93 \\
\hline
\end{tabular}

first-order shear deformation theory overestimates the first fundamental natural frequency deterministic and mean values by about $8 \%$. This difference is due to the difference in the number of degrees of freedom. As far as uncertainty is concerned, for the considered cases, $\Omega^{*}$ is not very sensitive to uncertainty in the material parameters. The percentage coefficient of variation of $\Omega^{*}$ is, at best, equal to three for a percentage coefficient of variation $(\mathrm{CV} \%)$ in the material elastic properties equal to 10. Material properties modelled via a Gaussian or a Weibull PDF yields similar stochastic results, unless small fractile values are desired. A CV\% equal to 2.88 in the geometrical parameters yield a percentage coefficient of variation of $\Omega^{*}$ equal to about five. A correlation between the thickness of the plate and $\Omega^{*}$ is present: the thicker the plate, the smaller the coefficient of variation.

\section{Acknowledgements}

This research is supported by the Ministère de la Culture, de l'Enseignement Supérieuer et de la Recherche of Luxembourg under contract R\&D BFR07/136-LB and two regional projects Piemonte 2004: E40 and E59. Finally, the authors would like to sincerely thank the reviewers for their precious comments. 


\section{References}

[1] H.I. Anderson, Metropolis, Monte Carlo and the MANIAC, Los Alamos Science 14 (1986), 96-108.

[2] M. Aydogdu and T. Timarci, Vibration analysis of cross-ply laminated square plates with general boundary conditions, Composites Science and Technology 63 (2003), 1061-1070.

[3] P.A. Blelloch, Direct correlation of test-analysis cross-orthogonality, Journal of Analytical and Experimental Modal Analysis 8 (1993), 247-255.

[4] P. Caravani and W.T. Thomson, Frequency response of a dynamic system with statistical damping, AIAA Journal 11 (1973), $170-173$.

[5] E. Carrera, $C_{z}^{0}$ requirements for the two dimensional analysis of multilayered structures, Composite Structures 37 (1997), 373-383.

[6] E. Carrera, Historical review of zig-zag theories for multilayered plates and shells, Applied Mechanics Review 56 (2003), 287-308.

[7] E. Carrera, A study of transverse normal stress effect on vibration of multilayered plates and shells, Journal of Sound and Vibration 225 (1999), 803-829.

[8] E. Carrera, Theories and finite elements for multilayered plates and shells: a unified compact formulation with numerical assessment and benchmarking, Archives of Computational Methods in Engineering 10 (2003), 215-296.

[9] A.L. Cauchy, Sur l'équilibre et le mouvement d'une plaque solide, Exercises de Matematique 3 (1828), 328-355.

[10] G.S. Fishman, Monte Carlo: concepts algorithms and applications, Springer, New York, 1996.

[11] M. Ganapathi and D.P. Makhecha, Free vibration analysis of multi-layered composite laminates based on an accurate higher-order theory, Composits: Part B Engineering 32 (2001), 535-543.

[12] G. Giunta and A. Calvi, Application of higher order derivatives to structural probabilistic analysis, ESA TEC-MCS report, The Netherlands, October 2004.

[13] S.S. Isukapalli and P.G. Georgopoulos, Computational Methods for Sensitivity and Uncertainty Analysis for Environmental and Biological Models, National Exposure Research Laboratory, U.S. Environmental Protection Agency, EPA/600/R-01-068, 2001.

[14] H.K. Jeong and R.A. Shenoi, Reliability analysis of mid-plane symmetric laminated plates using direct simulation method, Composite Structures 43 (1998), 1-13.

[15] M.V. Kalos and P.A. Whitlock, Monte Carlo Methods, volume I: basics, John Wiley and Sons, New York, 1986.

[16] T. Kant and K. Swaminathan, Analytical solutions for free vibration of laminated composite and sandwich plates based on higher-order refined theory, Composite Structures $\mathbf{5 3}$ (2001), 73-85.

[17] A.A. Khdeir and J.N. Reddy, Free vibrations of laminated composite plates using second order shear deformation theory, Computers and Structures 71 (1999), 617-626.

[18] G. Kirchhoff, Über das Gleichgewicht und die Bewegung einer elastishen Sceibe, J Reine Angew Math 40 (1850), 51-88.

[19] H. Matsunaga, Vibration and stability of cross-ply laminated composites plates according to a global higher-order plate theory, Composite Structures 48 (2000), 231-244.

[20] M.D. McKay, R.J. Beckman and W.J. Conover, A comparison of three methods for selecting values of input variables in the analysis of output from a computer code, Technometrics 21 (1979), 239-245.

[21] A. Messina, Two generalized higher order theories in free vibration studies of multilayered plates, Journal of Sound and Vibration 242 (2001), 125-150.

[22] N. Metropolis, The beginning of the Monte Carlo method, Los Alamos Science Special Issue 15 (1987), 125-130.

[23] N. Metropolis and S. Ulam, The Monte Carlo method, Journal of the American Statistical Association 44 (1949), $335-341$.

[24] E. Mindlin, Influence of the rotatory inertia and shear in flexural motions of isotropic elastic plates, Journal of Applied Mechanics 18 (1951), 1031-1036.

[25] M. Murakami, Laminated composites plate theory with improved in-plane response, Journal of Applied Mechanics 53 (1986), $661-666$.

[26] H. Nakayasu and Z. Maekawa, A comparative study of failure criteria in probabilistic fields and stochastic failure envelopes of composite materials, Reliability Engineering and System Safety 56 (1997), 209-220.

[27] A.K. Noor, Free vibration of multilayered composite plates, AIAA Journal 11 (1973), 1038-1039.

[28] S.D. Poisson, Mémoire sur l'équilibre et le mouvement des corps élastique, Mémoires de l'Académie des Sciences de Paris 8 (1829), 357-570.

[29] G.S. Fishman, Polymer matrix composites: Guidelines for characterization of structural materials, Composite materials handbook, MIL-HDBK-17-1F, Department of Defence, Washington, USA, 2002.

[30] H.I. Pradlwarter and G.I. Schuëller, Assessment of low probability events of dynamical systems by controlled Monte Carlo simulation, Probabilistic Engineering Mechanics 14 (1999), 213-227.

[31] C. Proppe and H.I. Pradlwarter and G.I. Schuëller, Equivalent linearization and Monte Carlo simulation in stochastic dynamics, Probabilistic Engineering Mechanics 18 (2003), 1-15.

[32] B.N. Raj and N.G.R. Iyengar and D. Yadav, Response of composite plates with random material properties using FEM and Monte Carlo simulation, Advanced Composites Materials 7 (1998), 219-237.

[33] J.N. Reddy, A simple higher order theory for laminated composites plates, Journal of Applied Mechanics 51 (1984), 745-752.

[34] E. Reissner, On a certain mixed variational theorem and a proposed Application, International Journal of Numerical Methods in Engineering 20 (1984), 1366-1368

[35] E. Reissner, The effect of transverse shear deformation on the bending of elastic plates, Journal of Applied Mechanics 12 (1945), 69-76.

[36] R.Y. Rubinstein, Simulation and the Monte Carlo Method, John Wiley and Sons, New York, 1981.

[37] A. Shaker, W.G. Abdelrahman, M. Tawfik and E. Sadek, Stochastic finite element analysis of the free vibration of laminated composite plates, Computational Mechanics 41 (2008), 493-501.

[38] M. Shinozuka, Monte Carlo solution of structural dynamics, Department of civil engineering and engineering mechanics, Columbia University, 1947.

[39] G.I. Schuëller, On the treatment of uncertainties in structural mechanics and analysis, Computers and Structures 85 (2007), $235-243$. 
[40] G.I. Schuëller, Computational stochastic mechanics - recent advances, Computers and Structures 79 (2001), $2225-2234$.

[41] G.I. Schuëller, Uncertainty \& Reliability Analysis of Structural Dynamical Systems, in: III European Conference on Computational Mechanics Solids, Structures and Coupled Problems in Engineering: Book of Abstracts, Springer, The Netherlands, 2006, p. 34.

[42] B.N. Singh, D. Yadav and N.G.R. Iyengar, Free vibration of composite cylindrical panels with random material properties, Composite Structures $\mathbf{5 8}$ (2002), 435-442.

[43] B.N. Singh, D. Yadav and N.G.R. Iyengar, Free vibration of laminated spherical panels with random material properties, Journal of Sound and Vibration 244 (2001), 321-338.

[44] B.N. Singh, D. Yadav and N.G.R. Iyengar, Natural frequencies of composite plates with random material properties using higher-order shear deformation theory, International Journal of Mechanical Sciences 43 (2001), 2193-2214.

[45] K.P. Soldatos, A Transverse Shear Deformation Theory for Homogeneous Monoclinic Plates, Acta Mechanica 94 (1992), $195-220$.

[46] S. Sriramula and M.K. Chryssanthopoulos, Probabilistic models for spatially varying mechanical properties of in-service GFRP cladding panels, Journal of Composites for Construction 13 (2009), 159-167.

[47] S. Sriramula and M.K. Chryssanthopoulos, Quantification of uncertainty modelling in stochastic analysis of FRP composites, Composites: Part A 40 (2009), 1673-1684.

[48] M. Touratier, An efficient standard plate theory, International Journal of Engineering Science 29 (1991), 901-916.

[49] D. Yadav and N. Verma, Free vibration of composite circular cylindrical shells with random material properties. Part I: General theory, Composite Structures 41 (1998), 331-338.

[50] D. Yadav and N. Verma, Free vibration of composite circular cylindrical shells with random material properties. Part II: Applications, Composite Structures 51 (2001), 371-380. 

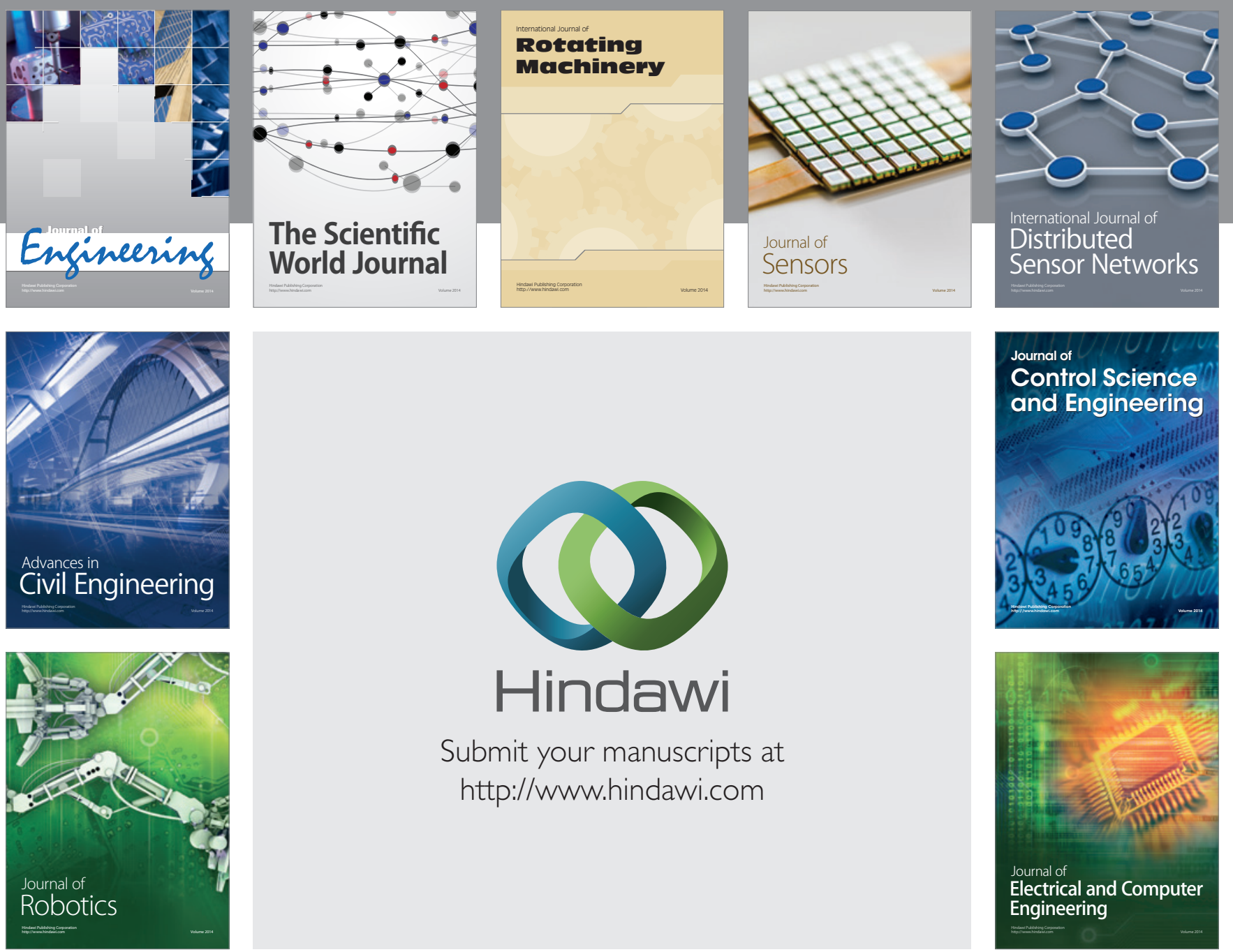

Submit your manuscripts at

http://www.hindawi.com
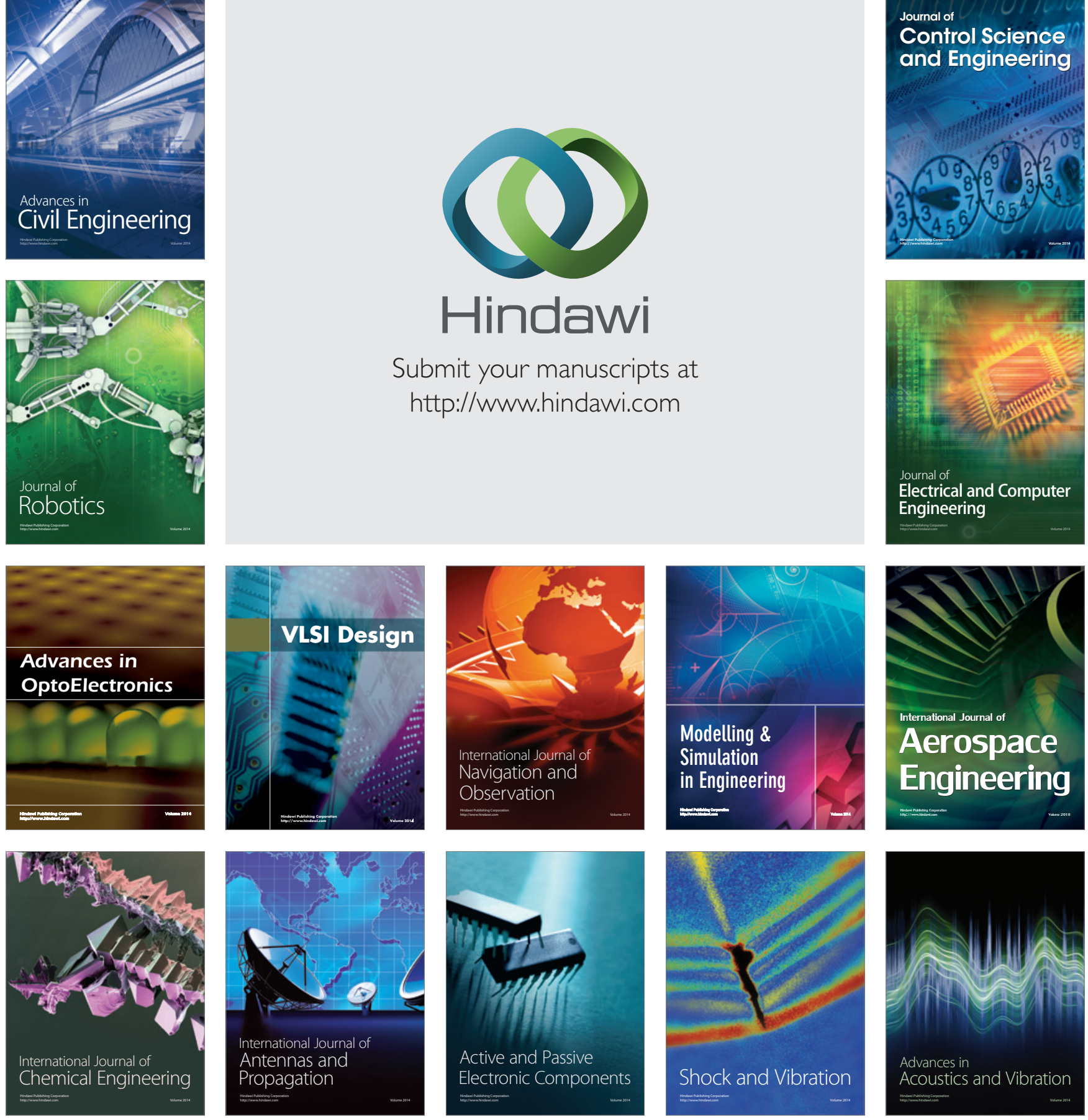